\title{
THE MILNOR NUMBER OF FUNCTIONS ON SINGULAR HYPERSURFACES
}

\author{
MARIUSZ ZAJ A̧ C \\ Institute of Mathematics, Warsaw University of Technology \\ Pl. Politechniki 1, 00-661 Warszawa, Poland \\ E-mail: zajac@plwatu21.bitnet
}

\begin{abstract}
The behaviour of a holomorphic map germ at a critical point has always been an important part of the singularity theory. It is generally known (cf. [5]) that we can associate an integer invariant - called the multiplicity - to each isolated critical point of a holomorphic function of many variables. Several years later it was noticed that similar invariants exist for function germs defined on isolated hypersurface singularities (see [1]). The present paper aims to show a simple approach to critical points of maps defined on the $A_{k}$-type singular hypersurfaces. After some changes it can probably be adopted to other isolated hypersurface singularities.
\end{abstract}

Acknowledgements. The author wishes to thank the Mathematical Research Institute in the Netherlands and the University of Utrecht for the invitation and hospitality, and Professor J. H. M.Steenbrink, who supervised this research project, for suggesting the problem and many stimulating discussions.

1. Let $\mathcal{O}_{n}$ denote the ring of germs of holomorphic functions $f:\left(\mathbf{C}^{n}, 0\right) \rightarrow(\mathbf{C}, 0)$.

Definition. The multiplicity of the critical point of $f$ at zero is $\mu(f):=\operatorname{dim}_{\mathbf{C}} \mathcal{O}_{n} / J_{f}$, where $J_{f}=\left\langle\partial f / \partial z_{1}, \ldots, \partial f / \partial z_{n}\right\rangle_{\mathcal{O}_{n}}$ is the Jacobian ideal of $f$.

The number $\mu(f)$ is also called the Milnor number of $f$, because it was first introduced by J. Milnor in 1968 [4].

Proposition. Let $f \in \mathcal{O}_{n}$ have an isolated critical point at zero and let $f(0)=0$. Then $\mu(f)$ is finite and the preimage of each sufficiently small non-zero complex number intersects a small open disk in a smooth manifold, which is homotopy equivalent to a bouquet of $\mu(f)(n-1)$-dimensional spheres.

$$
\left(\exists \varepsilon, \delta \in \mathbf{R}_{+}\right)(\forall t \in \mathbf{C}) \quad 0<|t|<\varepsilon \Rightarrow f^{-1}(t) \cap D_{\delta} \sim \bigvee_{\mu(f)} S^{n-1}
$$

1991 Mathematics Subject Classification: Primary 32S25.

The paper is in final form and no version of it will be published elsewhere. 
For a proof the reader is referred to Milnor's original article [4] as well as [3] and [5] - a survey article dealing with various definitions of multiplicity.

2. Let $\Omega^{i}(1 \leq i \leq n)$ denote the $\mathcal{O}_{n}$-module of germs of holomorphic $i$-forms at 0 . The explicit expressions for the most relevant cases: $i=n-1$ and $i=n$ are

$$
\Omega^{n}=\left\{g \cdot d z_{1} \wedge \ldots \wedge d z_{n} \mid g \in \mathcal{O}_{n}\right\}
$$

and

$$
\Omega^{n-1}=\left\{\sum_{k=1}^{n} g_{k} \cdot d z_{1} \wedge \ldots \wedge \widehat{d z_{k}} \wedge \ldots \wedge d z_{n} \mid g_{k} \in \mathcal{O}_{n}\right\}
$$

where the hat over $d z_{k}$ means "skip". Moreover, for all $f \in \mathcal{O}_{n}$ we have

$$
d f=\sum_{i=1}^{n} \frac{\partial f}{\partial z_{i}} \cdot d z_{i}
$$

Hence

$$
d f \wedge\left(\sum_{k=1}^{n} g_{k} \cdot d z_{1} \wedge \ldots \wedge \widehat{d z_{k}} \wedge \ldots \wedge d z_{n}\right)=\left(\sum_{k=1}^{n}(-1)^{k-1} g_{k} \cdot \frac{\partial f}{\partial z_{k}}\right) \cdot d z_{1} \wedge \ldots \wedge d z_{n}
$$

and denoting $d f \wedge \Omega^{n-1}:=\left\{d f \wedge \omega \mid \omega \in \Omega^{n-1}\right\}$, we obtain immediately

$$
\Omega^{n} /\left(d f \wedge \Omega^{n-1}\right) \cong \mathcal{O}_{n} / J_{f} \quad \text { and } \quad \operatorname{dim}_{\mathbf{C}} \Omega^{n} /\left(d f \wedge \Omega^{n-1}\right)=\mu(f) .
$$

ExAmple 1. We shall find the Milnor number of the function $f: \mathbf{C}^{2} \rightarrow \mathbf{C}$ given by

$$
f(z)=z_{1}^{e_{1}}+z_{2}^{e_{2}}
$$

where $e_{1}, e_{2} \geq 2$. Computing the Jacobian ideal gives us at once

$$
\mu(f)=e_{1} e_{2}-e_{1}-e_{2}+1=\left(e_{1}-1\right)\left(e_{2}-1\right),
$$

but the result could also be obtained by looking at fibres. Our function is quasi-homogeneous, hence $f^{-1}\left(t_{1}\right)$ is homeomorphic to $f^{-1}\left(t_{2}\right)$, for all $t_{1} \neq 0 \neq t_{2}$. We can therefore consider the fibre $F:=f^{-1}(1)$. Let $\xi_{1}=\exp \left(2 \pi i / e_{1}\right), \xi_{2}=\exp \left(2 \pi i / e_{2}\right)$ and put

$$
\begin{array}{ll}
P_{r}=\left(\xi_{1}^{r}, 0\right) & \text { for } r=0, \ldots, e_{1}-1, \\
Q_{s}=\left(0, \xi_{2}^{s}\right) & \text { for } s=0, \ldots, e_{2}-1 .
\end{array}
$$

Given $r$ and $s$, there is an arc $\gamma_{r, s} \subset F$ between $P_{r}$ and $Q_{s}$ described by

$$
\gamma_{r, s}:[0,1] \rightarrow F, \quad t \mapsto\left(\left(1-t^{e_{2}}\right)^{1 / e_{1}} \cdot \xi_{1}^{r}, t \cdot \xi_{2}^{s}\right) .
$$

It can be shown that the graph $G:=\bigcup_{r, s} \gamma_{r, s}$ is a retract of $F$ (see [5], p. 434). Moreover $G$ is homotopy equivalent to a bouquet of $\left(e_{1}-1\right)\left(e_{2}-1\right)$ circles.

3. For a fixed integer $k>1$ we shall consider the following singular hypersurface

$$
X:=\left\{\left(z_{1}, z_{2}, z_{3}\right) \in \mathbf{C}^{3} \mid z_{1}^{k}=z_{2} z_{3}\right\} .
$$

Denote also $X^{\prime}:=X \backslash\{(0,0,0)\}$.

Proposition. $X^{\prime}$ is a two-dimensional complex manifold.

Proof. Observe that $X^{\prime}=U_{1} \cup U_{2}$, where

$$
U_{1}=\left\{\left(z_{1}, z_{2}, z_{3}\right) \in X \mid z_{2} \neq 0\right\}, \quad U_{2}=\left\{\left(z_{1}, z_{2}, z_{3}\right) \in X \mid z_{3} \neq 0\right\} .
$$


Both sets $U_{i}$ are homeomorphic to $\mathbf{C} \times(\mathbf{C} \backslash\{0\})$; the homeomorphisms can be

$$
\begin{array}{rlrl}
h_{1}: U_{1} \rightarrow \mathbf{C} \times(\mathbf{C} \backslash\{0\}), & & \left(z_{1}, z_{2}, z_{3}\right) \mapsto\left(z_{1}, z_{2}\right), \\
h_{2}: U_{2} \rightarrow \mathbf{C} \times(\mathbf{C} \backslash\{0\}), & \left(z_{1}, z_{2}, z_{3}\right) \mapsto\left(z_{1}, z_{3}\right) .
\end{array}
$$

Moreover, the transition function

$$
h_{2} \circ h_{1}^{-1}:(\mathbf{C} \backslash\{0\})^{2} \rightarrow(\mathbf{C} \backslash\{0\})^{2}, \quad(s, t) \mapsto\left(s, s^{k} / t\right),
$$

is holomorphic.

Proposition. The mapping $m: \mathbf{C}^{2} \rightarrow X$ defined by $m(s, t):=\left(s t, s^{k}, t^{k}\right)$ induces a holomorphic covering $m^{\prime}: \mathbf{C}^{2} \backslash\{(0,0)\} \rightarrow X^{\prime}$. The preimage of any $x^{\prime} \in X^{\prime}$ consists of $k$ points of the form $\left(\xi^{i} s, \xi^{-i} t\right)$, where $0 \leq j \leq k-1$ and $\xi=\exp (2 \pi i / k)$.

Corollary. Every holomorphic function $f^{\prime}: X^{\prime} \rightarrow \mathbf{C}$ comes from a holomorphic function $\tilde{f}: \mathbf{C}^{2} \backslash\{(0,0)\} \rightarrow \mathbf{C}$ satisfying $\tilde{f}\left(\xi s, \xi^{-1} t\right)=\tilde{f}(s, t)$.

The following diagram is then commutative

$$
\begin{aligned}
\mathbf{C}^{2} \backslash\{(0,0)\} & \\
m & \downarrow \searrow_{f} \\
X^{\prime} & \stackrel{f^{\prime}}{\longrightarrow} \mathbf{C}
\end{aligned}
$$

A well-known theorem of Hartogs implies that every holomorphic function defined on $\mathbf{C}^{2} \backslash\{(0,0)\}$ extends to $\mathbf{C}^{2}$. Hence the preceding considerations result in the following

Proposition. If $f: X \rightarrow \mathbf{C}$ is a continuous function such that $\left.f\right|_{X^{\prime}}$ is holomorphic then there exists a holomorphic function $\tilde{f}: \mathbf{C}^{2} \rightarrow \mathbf{C}$ making the following diagram commutative:

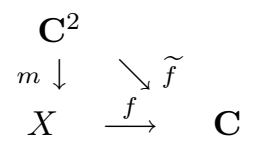

$\widetilde{f}$ is invariant under the action of the covering group, i.e.

$$
\tilde{f}\left(\xi s, \xi^{-1} t\right)=\tilde{f}(s, t) .
$$

On the other hand, if $\tilde{f}=\sum a_{p q} s^{p} t^{q}(1)$ is equivalent to

$$
a_{p q}=0 \quad \text { if } p \not \equiv q(\bmod k) .
$$

Therefore if $\tilde{f}: \mathbf{C}^{2} \rightarrow \mathbf{C}$ satisfies (1) then all non-zero components $a_{p q} s^{p} t^{q}$ can be written as $a \cdot\left(s^{k}\right)^{m_{1}} \cdot(s t)^{m_{2}}$ or $a \cdot\left(t^{k}\right)^{m_{1}} \cdot(s t)^{m_{2}}$. In this manner $\tilde{f}$ induces a function $f: X \rightarrow \mathbf{C}$. If $\tilde{f}$ is holomorphic then $f$ is continuous on $X$ and holomorphic on $X^{\prime}$. In the sequel we shall sometimes identify corresponding functions $f: X \rightarrow \mathbf{C}$ and $\tilde{f}: \mathbf{C}^{2} \rightarrow \mathbf{C}$.

4. Let $f: X \rightarrow \mathbf{C}$ and $\tilde{f}: \mathbf{C}^{2} \rightarrow \mathbf{C}$ be corresponding functions in the sense of the above diagram. If $f((0,0,0))=0$ then for $t \neq 0$

$$
\left.m\right|_{\tilde{f}^{-1}(t)}: \tilde{f}^{-1}(t) \rightarrow f^{-1}(t)
$$

is an unramified covering of degree $k$. If we define $F_{t}$ as we have above for functions $\mathbf{C}^{2} \rightarrow \mathbf{C}$ then we can easily see that

$$
\chi\left(\tilde{F}_{t}\right)=k \cdot \chi\left(F_{t}\right),
$$


where $\chi$ denotes the Euler characteristic.

THEOREM. If 0 is an isolated critical point of $\tilde{f}$ then for all sufficiently small $t \neq 0$

$$
F_{t} \sim \bigvee_{\mu_{X}(f)} S^{1}
$$

where

$$
\mu_{X}(f)=\frac{\mu(\tilde{f})-1}{k}+1
$$

Pr o of. The fibre $\tilde{F}_{t}$ is a Riemann surface. Viewed as a subset of $\mathbf{C P}^{2}$ it becomes a compact Riemann surface (a sphere with handles) with a finite number of disks removed. Using properties of coverings we see that $F_{t}$ is also a surface of this type, hence it is homotopy equivalent to a bouquet of a certain number of circles. We know that

$$
\tilde{F}_{t} \sim \bigvee_{\mu(\tilde{f})} S^{1} \quad \text { and } \quad \chi\left(\tilde{F}_{t}\right)=\mu(\tilde{f})-1
$$

Equation (2) gives now the required result.

ExAmple 2. Consider $f: X \rightarrow \mathbf{C}$ induced by

$$
\tilde{f}(s, t)=s^{k \cdot e_{1}}+t^{k \cdot e_{2}},
$$

where $e_{1}, e_{2} \geq 2$. The above theorem yields together with Example 1

$$
\mu_{X}(f)=\frac{\left(k e_{1}-1\right)\left(k e_{2}-1\right)-1}{k}+1=k \cdot e_{1} e_{2}-e_{1}-e_{2}+1 .
$$

On the other hand, the reader can check that the graph $m(\Gamma)$ is homotopy equivalent to a bouquet of

$$
\mu=\left(e_{1}-1\right)\left(e_{2}-1\right)+(k-1) \cdot e_{1} e_{2}
$$

circles. Obviously $\mu_{X}(f)=\mu$.

5. Although the Jacobian ideal of a function $f: X \rightarrow \mathbf{C}$ cannot be defined in the previous way, we can proceed using the language of differential forms. Let $\mathcal{F}, \mathcal{G}^{1}, \mathcal{G}^{2}$ be the sheaves of holomorphic functions, 1 -forms and 2-forms on $X^{\prime}$, respectively. If $j: X^{\prime} \hookrightarrow X$ is the natural inclusion, then the stalks

$$
\mathcal{O}_{X}:=\left(j_{*} \mathcal{F}\right)_{(0,0,0)}, \quad \Omega_{X}^{1}:=\left(j_{*} \mathcal{G}^{1}\right)_{(0,0,0)} \quad \text { and } \quad \Omega_{X}^{2}:=\left(j_{*} \mathcal{G}^{2}\right)_{(0,0,0)}
$$

are analogues of the ring of germs of holomorphic functions and the modules of germs of holomorphic 1-forms and 2-forms. This enables us to define the algebraic multiplicity in the current context by the formula

$$
\mu_{a}(f):=\operatorname{dim}_{\mathbf{C}} \Omega_{X}^{2} /\left(d f \wedge \Omega_{X}^{1}\right) .
$$

Also differential forms on $X$ are induced by those forms on $\mathbf{C}^{2}$ which satisfy certain invariance conditions. In fact $\Omega_{X}^{2}$ is generated as an $\mathcal{O}_{X}$-module by the form induced by $d s \wedge d t$, while $\Omega_{X}^{1}$ needs four generators: $s^{k-1} \cdot d s, t \cdot d s, s \cdot d t$ and $t^{k-1} \cdot d t$. Therefore we have

$$
\mu_{a}(f)=\operatorname{dim}_{\mathbf{C}} \mathcal{O}_{X} /\left\langle s^{k-1} \frac{\partial \tilde{f}}{\partial t}, t \frac{\partial \tilde{f}}{\partial t}, s \frac{\partial \tilde{f}}{\partial s}, t^{k-1} \frac{\partial \tilde{f}}{\partial s}\right\rangle_{\mathcal{O}_{X}}
$$


EXAmple 3. Let us return to the function $f: X \rightarrow \mathbf{C}$ induced by

$$
\tilde{f}(s, t)=s^{k \cdot e_{1}}+t^{k \cdot e_{2}}
$$

where $e_{1}, e_{2} \geq 2$. Now

$$
\mu_{a}(f)=\operatorname{dim}_{\mathbf{C}} \mathcal{O}_{X} /\left\langle(s t)^{k-1} \cdot\left(t^{k}\right)^{e_{2}-1}, t^{k \cdot e_{2}}, s^{k \cdot e_{1}},(s t)^{k-1} \cdot\left(s^{k}\right)^{e_{1}-1}\right\rangle_{\mathcal{O}_{X}} .
$$

An explicit calculation shows that $\mu_{a}(f)=k \cdot e_{1} e_{2}-e_{1}-e_{2}+1$.

\section{References}

[1] A. Dimca, Function germs defined on isolated hypersurface singularities, Compositio Math. 53 (1984), 245-258.

[2] A. G. Kouchnirenko, Polyèdres de Newton et nombres de Milnor, Invent. Math. 32 (1976), 1-31.

[3] V. P. Palamodov, Multiplicity of holomorphic mappings, Funct. Anal. Appl. 1 (1967), 218-266.

[4] J. Milnor, Singular Points of Complex Hypersurfaces, Ann. of Math. Stud. 61, Princeton Univ. Press, 1968.

[5] P. Orlik, The multiplicity of a holomorphic map at an isolated critical point, in: P. Holm (ed.), Real and Complex Singularities, Proc. Nordic Summer School/NAVF, Oslo 1976, Sijthoff \& Noordhoff, 1977, 405-474. 